\title{
Pulse Roguing Strategy in a Pine Wilt Disease Epidemic Model with General Nonlinear Incidence Rate
}

\author{
Quanben Sun, Wugui Chen, Zhicai Guo, Weiwei Ji, Jianping Wang* \\ The Department of Basic Course Education, Ji'an College, Ji'an, China \\ Email: ^jpwmath@126.com
}

How to cite this paper: Sun, Q.B.., Chen, W.G., Guo, Z.C., Ji, W.W. and Wang, J.P. (2020) Pulse Roguing Strategy in a Pine Wilt Disease Epidemic Model with General Nonlinear Incidence Rate. Journal of Applied Mathematics and Physics, 8, 2943-2953. https://doi.org/10.4236/jamp.2020.812217

Received: November 24, 2020

Accepted: December 18, 2020

Published: December 21, 2020

Copyright $\odot 2020$ by author(s) and Scientific Research Publishing Inc. This work is licensed under the Creative Commons Attribution International License (CC BY 4.0).

http://creativecommons.org/licenses/by/4.0/

\begin{abstract}
In this study, we investigate a pine wilt transmission model with general nonlinear incidence rates and time-varying pulse roguing. Using the stroboscopic map and comparison theorem, we proved that the disease-free equilibrium is global attractive determined by the basic reproduction number $R_{1}<1$, and in such a case, the endemic equilibrium does not exist. The disease uniformly persists only if $R_{2}>1$.
\end{abstract}

\section{Keywords}

Pine Wilt Disease, Pulse Roguing, General Nonlinear Incidence, Permanence

\section{Introduction}

Pine wilt disease (PWD) is a dramatic disease of pine caused by the pinewood nematode (Bursaphelenchus xylophilus), which constitutes a major threat to forest ecosystems worldwide, from both the economical point of view and the environmental perspective [1]-[8]. PWD is vector-born disease which is transmitted by pine sawyer beetle. The healthy host pine trees are usually killed within a few months by nematodes [4]. The first visible symptom is lack of resin exudation of bark wounds. The foliage becomes light grayish green, then becomes yellow, and finally it becomes reddish brown. The pine trees affected by pine wilt disease totally lack resin and wood becomes dry [9]. During the 20th century, the greatest losses have occurred due to pine wilt disease and it had a devastating impact, spreading among highly susceptible Japanese Black Pine and Japanese red pine forest. In addition, it spread from Japan to neighboring East Asian countries such as China and Korea in 1982 and 1988, respectively, and it was 
detected in Portugal in 1999 [10]. Pine wilt disease is a grave threat to forests worldwide and will potentially become a global epidemic.

Mathematical models play an important role in understanding the epidemiology of pine wilt diseases, control disease, and gain a deeper insight into the disease transmission dynamics in a population. There are many authors that establish mathematical models to describe the transmission of plant disease. In [11], Lee introduced a mathematical model on pine wilt disease and discussed the stability of pine wilt disease with optimal control. X. Y. Shi and G. H. Song [12] formulated a four-dimensional system involving only the pine and Monochamus alternatus and analyzed global dynamics of the model. The model is as follows:

$$
\left\{\begin{array}{l}
\frac{\mathrm{d} S}{\mathrm{~d} t}=b-\beta \frac{Y}{X+Y} S-\alpha S, \\
\frac{\mathrm{d} I}{\mathrm{~d} t}=\beta \frac{Y}{X+Y} S-\delta I, \\
\frac{\mathrm{d} X}{\mathrm{~d} t}=a-k \frac{I}{S+I} X-\mu X, \\
\frac{\mathrm{d} Y}{\mathrm{~d} t}=k \frac{I}{S+I} X-\mu Y .
\end{array}\right.
$$

Lee and Lashari introduced a mathematical model that incorporated the exposed class in the pine tree population and made a detailed discussion on the stability and optimal control. Ozair presented a mathematical model of the dynamics of pine wilt disease by dividing the host pine trees and vector beetles into susceptible and infected classes with nonlinear incidence and horizontal transmission [13]. The classical epidemiological models are developed by the assumption of bilinear incidence rate $\beta S I$ and the standard incidence rate $\frac{\beta S I}{N}$. Capasso and Serio [14] introduced a saturated incidence rate $g(I) S$ in an epidemic model. Liu [15] [16] investigated nonlinear incidence rates of the form $\beta S^{p} I^{q}$, and proposed a Holling-type nonlinear incidence function given by $\frac{\beta S I^{p}}{1+\alpha I^{p}}$. A variety of nonlinear incidence rates has been utilized in epidemic models [17]-[22]. In the real world, farmers' experiences include replanting, and rouging diseased plants to control the disease spread. What's more, the disease transmission is infected by season and temperature, et al. To address the time-varying impulse of dynamic model with general nonlinear, incidence rate has been analyzed.

The paper is organized as follows. In the next section, an impulsive epidemic model with general nonlinear incidence rate is formulated. In Sections 3 and 4, we proved the sufficient condition for the global attractivity of the disease-free equilibrium and the permanence of the model, respectively.

\section{Model Formulation and Preliminary}

In this section, we introduced a more advanced model that the pine trees population is subdivided into three groups: susceptible pine trees $S(t)$, asympto- 
matic carrier pine trees $A(t)$ and infected pine trees $I(t)$. We assume that roguing asymptomatic carrier pine trees and infected pine trees at the same time.

Additionally, we consider the disease mortality in the symptomatic pine trees population and assume that roguing asymptomatic and symptomatic group at different rate. Motivated by the above reasons, we build the epidemic model with general nonlinear incidence rate and time-varying impulse as follows:

$$
\left\{\begin{array}{l}
\frac{\mathrm{d} S(t)}{\mathrm{d} t}=\alpha(K-S(t)-A(t)-I(t))-f(t, S, A)-g(t, S, I)-\gamma S(t), \\
\frac{\mathrm{d} A(t)}{\mathrm{d} t}=f(t, S, A)-\gamma E(t), \\
\frac{\mathrm{d} I(t)}{\mathrm{d} t}=g(t, S, I)-\gamma I(t)-\mu I(t), \\
S\left(t^{+}\right)=S(t), \\
\left.A\left(t^{+}\right)=\left(1-p_{k}\right) A(t),\right\} t=t_{k}, k \in N . \\
I\left(t^{+}\right)=\left(1-\theta_{k}\right) I(t),
\end{array}\right\} t \neq t_{k}, k \in N,
$$

The following assumptions are satisfied for the model.

(H1) $S(t), A(t)$ and $I(t)$ are left continuous for $\left[t_{0},+\infty\right)$, that is $S(t)=\lim _{h \rightarrow 0^{+}} S(t-h), A(t)=\lim _{h \rightarrow 0^{+}} A(t-h), \quad I(t)=\lim _{h \rightarrow 0^{+}} I(t-h)$.

(H2) There is a maximum pine trees population size $K>0$. Replant the pine trees population at a rate $\alpha>0$.

(H3) $\gamma>0$ denotes the natural death rate of pine trees, $\mu \geq 0$ is the disease induced death rate.

(H4) $\theta_{k}$ is symptomatic pine wilt disease at fixed time $t=t_{k}$, and $\theta_{k}=\theta_{q+k}$.

(H5) $t_{k}$ is pulse time. There exist a positive integer $q$ and a positive $\omega$ such that $t_{k+q}=t_{k}+\omega$ for all $k \in N$.

(H6) $f(t, S, A)$ and $g(t, S, I)$ are both piecewise continuous and positive $\omega$-periodic functions, which means incidence rates about group $A$ and $I$, respectively. The form of it is as follows:

$$
f(t, S, A)=\left\{\begin{array}{cl}
f_{1}(t, S, A), & t \in\left(t_{0}+n \omega, t_{1}+n \omega\right], \\
\vdots & \\
f_{q}(t, S, A), & t \in\left(t_{q-1}+n \omega, t_{q}+n \omega\right],
\end{array}\right.
$$

and

$$
g(t, S, I)=\left\{\begin{array}{cl}
g_{1}(t, S, I), & t \in\left(t_{0}+n \omega, t_{1}+n \omega\right], \\
\vdots & \\
g_{q}(t, S, I), & t \in\left(t_{q-1}+n \omega, t_{q}+n \omega\right],
\end{array}\right.
$$

for all nonnegative integer $n$, and $f_{i}(t, 0, A)=f_{i}(t, S, 0)=0$, $g_{i}(t, 0, I)=g_{i}(t, S, 0)=0$ for $i=1,2, \cdots, q$.

\section{The Disease-Free Periodic Solution Is Global Attractive}

From system (2), we can easily obtain that $\left(\frac{\alpha K}{\alpha+\gamma}, 0,0\right)$ is the disease-free 
periodic solution. To analyze the global attractiveness of the disease-free periodic solution, we give the hypothesis as follows:

(B1) There exist two piecewise continuous and positive $\omega$-periodic function $\xi_{i}(t), \beta_{i}(t)$, that is $\xi_{i}(t)=\xi_{i}(t+\omega), \beta_{i}(t)=\beta_{i}(t+\omega)$, for $i=1,2, \cdots, q$, such that $f_{i}(t, S, A) \leq \xi_{i}(t) S(t) A(t), \quad g_{i}(t, S, I) \leq \beta_{i}(t) S(t) I(t)$ for all $t \geq t_{0}$.

Let

$$
R_{1}=\frac{\frac{\alpha K}{\alpha+\gamma} \sum_{i=1}^{q} \int_{t_{i-1}}^{t_{i}} \psi_{i}(t) \mathrm{d} t}{\gamma \omega-\sum_{i=1}^{q} \ln \left(1-a_{i}\right)}
$$

and

$$
a_{i}=\min \left\{p_{i}, \theta_{i}\right\}, \psi_{i}(t)=\max \left\{\xi_{i}(t), \beta_{i}(t)\right\}, i=1,2, \cdots, q .
$$

It's easy to see that $\left(\frac{\alpha K}{\alpha+\gamma}, 0,0\right)$ is the disease-free periodic solution of system (1).

Theorem 1. If system (2) satisfies $R_{1}<1$ and the (B1), then the disease-free periodic solution is globally attractive.

Proof: Let $(S(t), A(t), I(t))$ be any solution of system (2), since $R_{1}<1$, we have

$$
\Omega \triangleq \exp \left[\sum_{i=1}^{q} \int_{t_{i-1}}^{t_{i}}\left[\psi_{i}(t)\left(\frac{\alpha K}{\alpha+\gamma}+\varepsilon_{1}\right)\right] \mathrm{d} t+\sum_{i=1}^{q} \ln \left(1-a_{i}\right)-\gamma \omega\right],
$$

where $\varepsilon_{1}>0$ is a sufficiently small number.

From the first equation of (2), we have $\frac{\mathrm{d} S(t)}{\mathrm{d} t} \leq \alpha K-\alpha S(t)-\gamma S(t)$. By the comparison theorem, for arbitrarily small number $\varepsilon_{1}$, there exists a positive constant $t^{\prime}\left(>t_{0}\right)$, such that

$$
S(t)<\frac{\alpha K}{\gamma}+\varepsilon_{1}, \text { for all } t \geq t^{\prime} .
$$

Therefore, let $\varepsilon_{1} \rightarrow 0$, we can obtain that $\lim _{t \rightarrow \infty}\left(S(t)-S^{*}(t)\right)=0$.

Next, from (5) and the second equation of system (2), for $t \in\left(t_{i-1}+n \omega, t_{i}+n \omega\right](i=1,2, \cdots, q)$ and $t \geq t^{\prime}$, we have

$$
\begin{aligned}
\frac{\mathrm{d} A(t)}{\mathrm{d} t}+\frac{\mathrm{d} I(t)}{\mathrm{d} t} & =g(t, S, A)+f(t, S, I)-\gamma(A(t)+I(t))-\mu I(t) \\
& \leq \xi_{i} S(t) A(t)+\beta_{i} S(t) I(t)-\gamma(A(t)+I(t)) \\
& \leq \psi_{i}\left(S^{*}(t)+\varepsilon_{1}\right)(A(t)+I(t))-\gamma(E(t)+I(t)) \\
& \leq\left[\psi_{i}(t)\left(\frac{\alpha K}{\gamma}+\varepsilon_{1}\right)-\gamma\right](E(t)+I(t)),
\end{aligned}
$$

where $\psi_{i}(t)(i=1,2, \cdots, q)$ is same as (3). Thus

$$
\begin{aligned}
& A(t)+I(t) \\
& \leq\left(A\left(\left(t_{i-1}+n \omega\right)^{+}\right)+I\left(\left(t_{i-1}+n \omega\right)^{+}\right)\right) \exp \int_{t_{i-1}+n \omega}^{t}\left[\psi_{i}(t)\left(\frac{\alpha K}{\alpha+\gamma}+\varepsilon_{1}\right)-\gamma\right] \mathrm{d} t \\
& =\left[\left(1-p_{i-1}\right) A\left(t_{i-1}+n \omega\right)+\left(1-\theta_{i-1}\right) I\left(t_{i-1}+n \omega\right)\right] \cdot \exp \int_{t_{i-1}+n \omega}^{t}\left[\psi_{i}(t)\left(\frac{\alpha K}{\gamma}+\varepsilon_{1}\right)-\gamma\right] \mathrm{d} t \\
& \leq\left(1-a_{i-1}\right)\left(A\left(t_{i-1}+n \omega\right)+I\left(t_{i-1}+n \omega\right)\right) \exp \int_{t_{i-1}+n \omega}^{t}\left[\psi_{i}(t)\left(\frac{\alpha K}{\alpha+\gamma}+\varepsilon_{1}\right)-\gamma\right] \mathrm{d} t,
\end{aligned}
$$


where $a_{i}(i=1,2, \cdots, q)$ is same as (3).

By the similar method, we get that for $t \in\left(t_{i-1}+n \omega, t_{i}+n \omega\right]$.

$A(t)+I(t)$

$\leq \prod_{j=1}^{i-1}\left(1-a_{j}\right)\left[A\left(\left(t_{0}+n \omega\right)^{+}\right)+I\left(\left(t_{0}+n \omega\right)^{+}\right)\right]$

$$
\cdot \exp \left\{\left(\frac{\alpha K}{\alpha+\gamma}+\varepsilon_{1}\right)\left[\int_{t_{0}+n \omega}^{t_{1}+n \omega} \psi_{1}(t) \mathrm{d} t+\cdots+\int_{t_{i-1}+n \omega}^{t} \psi_{i}(t) \mathrm{d} t\right]-\gamma\left(t-t_{0}-n \omega\right)\right\} \text {. }
$$

Especially, we obtain that

$$
\begin{aligned}
& A\left(\left(t_{0}+(n+1) \omega\right)^{+}\right)+I\left(\left(t_{0}+(n+1) \omega\right)^{+}\right) \\
\leq & \left(1-a_{q}\right)\left(A\left(t_{q}+n \omega\right)+I\left(t_{q}+n \omega\right)\right) \\
\leq & \prod_{i=1}^{q}\left(1-a_{i}\right)\left[A\left(\left(t_{0}+n \omega\right)^{+}\right)+I\left(\left(t_{0}+n \omega\right)^{+}\right)\right] \\
& \cdot \exp \left[\sum_{i=1}^{q} \int_{t_{i-1}}^{t_{i}}\left[\psi_{i}(t)\left(\frac{\alpha K}{\alpha+\gamma}+\varepsilon_{1}\right)\right] \mathrm{d} t-\gamma\left(t_{q}-t_{0}\right)\right] \\
= & {\left[A\left(t_{0}+n \omega\right)+I\left(t_{0}+n \omega\right)\right] } \\
& \cdot \exp \left[\sum_{t_{i-1}}^{t_{i}} \int_{t_{i-1}}^{t_{i}}\left[\psi_{i}(t)\left(\frac{\alpha K}{\alpha+\gamma}+\varepsilon_{1}\right)\right] \mathrm{d} t+\sum_{i=1}^{q} \ln \left(1-a_{i}\right)-\gamma \omega\right] \\
= & \Omega\left[A\left(\left(t_{0}+n \omega\right)^{+}\right)+I\left(\left(t_{0}+n \omega\right)^{+}\right)\right],
\end{aligned}
$$

when $t=t_{0}+(n+1) \omega$.

Then, for an any positive integer $s$, we obtain

$$
\begin{aligned}
& A\left(\left(t_{0}+(n+s) \omega\right)^{+}\right)+I\left(\left(t_{0}+(n+s) \omega\right)^{+}\right) \\
\leq & \Omega^{s}\left[A\left(\left(t_{0}+n \omega\right)^{+}\right)+I\left(\left(t_{0}+n \omega\right)^{+}\right)\right],
\end{aligned}
$$

It follows from (4) and (8), we have that

$$
A\left[\left(t_{0}+(n+s) \omega\right)^{+}\right]+I\left[\left(t_{0}+(n+s) \omega\right)^{+}\right] \rightarrow 0, \text { as } s \rightarrow+\infty
$$

From (7) and (8), we obtain that

$$
\lim _{t \rightarrow+\infty}(A(t)+I(t))=0
$$

that is, for any arbitrarily small number $\varepsilon_{1}$, there exists $t^{2}\left(>t^{1}\right)$, such that $A(t)+I(t)<\varepsilon_{1}$ for $t>t^{2}$. Then, from the first equation of system (2), we know that

$$
\begin{aligned}
\frac{\mathrm{d} S(t)}{\mathrm{d} t} & =\alpha(K-A(t)-I(t))-g(t, S, A)-f(t, S, I)-\gamma S(t) \\
& \geq \alpha\left(K-\varepsilon_{1}\right)-\left(\psi^{*} \varepsilon_{1}+\alpha+\gamma\right) S(t)
\end{aligned}
$$

for $t>t^{2}$, where $\psi^{*}=\max _{1 \leq i \leq q} \psi_{i}(t), t \in\left[t_{0}, t_{0}+\omega\right]$. Solving the differential inequality, we can obtain

$$
\begin{aligned}
S(t) & \geq \frac{\alpha\left(K-\varepsilon_{1}\right)}{\psi^{*} \varepsilon_{1}+\alpha+\gamma}+\left[S\left(t^{2}\right)-\frac{\alpha\left(K-\varepsilon_{1}\right)}{\psi^{*} \varepsilon_{1}+\alpha+\gamma}\right] \mathrm{e}^{-\left(t-t^{2}\right)\left(\psi^{*} \varepsilon_{1}+\alpha+\gamma\right)} \\
& \triangleq \tilde{S}(t), \text { for } t>t^{2} .
\end{aligned}
$$


Combining (5) with (12), we get that

$$
\tilde{S}(t) \leq S(t) \leq \frac{\alpha K}{\alpha+\gamma}+\varepsilon_{1}, \text { for }\left(t>t^{2}\right) .
$$

Because $\varepsilon_{1}$ is an arbitrary positive small number, (12) means that

$$
\lim _{t \rightarrow+\infty} S(t)=\frac{\alpha K}{\alpha+\gamma} .
$$

By (10) and (13), we know that the disease-free periodic solution $\left(\frac{\alpha K}{\alpha+\gamma}, 0,0\right)$ is global attractive.

\section{Permanence}

In this section, the sufficient conditions of system (2) for the permanence are obtained. At firstly, we give the hypothesis (B1) as following:

(B2) There exist two piecewise continuous and positive $\omega$-periodic functions $\varsigma_{i}(t), \eta_{i}(t)$, such that $f_{i}(t, S, A) \geq \varsigma_{i}(t) S(t) A(t), \quad g_{i}(t, S, I) \geq \eta_{i}(t) S(t) I(t)$, for all $t \geq t_{0}$.

Let

$$
R_{2}=\frac{\frac{\alpha K}{\alpha+\gamma} \sum_{i=1}^{q} \int_{t_{i-1}}^{t_{i}} \varphi_{i}(t) \mathrm{d} t}{(\mu+\gamma) \omega-\sum_{i=1}^{q} \ln \left(1-b_{i}\right)}
$$

and

$$
b_{i}=\max \left\{p_{i}, \theta_{i}\right\}, \varphi_{i}(t)=\min \left\{\varsigma_{i}(t), \eta_{i}(t)\right\}, i=1,2, \cdots, q .
$$

Theorem 2. The system (1) is permanence, when $R_{2}>1$ and the assumptions (B1) and (B2) hold.

Proof: We have $R_{2}>1$, there exists an arbitrarily small number $\varepsilon_{2}>0$ such that

$$
\Lambda \triangleq \prod_{i=1}^{q}\left(1-b_{i}\right) \exp \left[\left(\frac{\alpha\left(K-\varepsilon_{2}\right)}{\alpha+\gamma}-\varepsilon_{2}\right) \sum_{i=1}^{q} \int_{t_{i-1}}^{t_{i}} \varphi_{i}(t) \mathrm{d} t-(\mu+\gamma) \omega\right]>1 .
$$

For proving the conclusion, we prove the disease is uniformly weakly persistent, firstly. There exists a constant $\delta>0$, such that $\limsup _{t \rightarrow+\infty}(A(t)+I(t)) \geq \delta$. By contradiction, for $\varepsilon_{2}>0$, there exists $t^{2}>0$ such that $A(t)+I(t)<\varepsilon_{2}$ for $t>t^{2}$.

In view of the hypothesis (B1) and the first equation of model (2), we have

$$
\begin{aligned}
\frac{\mathrm{d} S(t)}{\mathrm{d} t} & =\alpha(K-S(t)-A(t)-I(t))-g(t, S, A)-f(t, S, I)-\gamma S(t) \\
& \geq \alpha\left(K-\varepsilon_{1}\right)-\left(\psi^{*} \varepsilon_{2}+\alpha+\gamma\right) S(t)
\end{aligned}
$$

for all $t>t^{3}$, where $\psi^{*}$ is defined in (10). Consider the following auxiliary system:

$$
\frac{\mathrm{d} y_{1}}{\mathrm{~d} t}=\alpha\left(K-\varepsilon_{2}\right)-\left(\psi^{*} \varepsilon_{2}+\alpha+\gamma\right) y_{1}(t) .
$$


$y_{1}(t)$ is the solution of system (16), it is easy to know that $y_{1}(t) \rightarrow \frac{\alpha\left(K-\varepsilon_{2}\right)}{\psi^{*} \varepsilon_{2}+\alpha+\gamma}$ as $t \rightarrow+\infty$. By the comparison theorem, we get $S(t) \geq y_{1}(t)$. Therefore, for any arbitrarily small $\varepsilon_{2}$, there have an integer $n^{*}>0$, such that

$$
S(t) \geq y_{1}(t) \geq \frac{\alpha\left(K-\varepsilon_{2}\right)}{\psi^{*} \varepsilon_{2}+\alpha+\gamma}-\varepsilon_{2}, \text { for } t>t^{3}+n^{*} \omega .
$$

For above $t^{2}+t^{\prime}$ and a positive integer $n_{1}$, we have $n_{1} \omega \geq t^{2}+t^{\prime}$. Therefore, for all $n \omega+t_{i-1}<t<n \omega+t_{i},\left(n \geq N_{1}, i=1,2, \cdots, q\right)$, according to the second equation of system (2) and (17), we get

$$
\begin{aligned}
& \frac{\mathrm{d} A(t)}{\mathrm{d} t}+\frac{\mathrm{d} I(t)}{\mathrm{d} t} \\
& =f(t, S, A)+g(t, S, I)-\gamma(A(t)+I(t))-\mu I(t) \\
& \geq \varsigma_{i}(t) S(t) A(t)+\eta_{i}(t) S(t) I(t)-\gamma(A(t)+I(t))-\mu I(t) \\
& \geq \varphi_{i}(t) S(t)(A(t)+I(t))-\gamma(A(t)+I(t))-\mu(A(t)+I(t)) \\
& \geq\left[\varphi_{i}(t)\left(\frac{\alpha\left(K-\varepsilon_{2}\right)}{\psi^{*} \varepsilon_{2}+\alpha+\gamma}-\varepsilon_{2}\right)-\gamma-\mu\right](A(t)+I(t))
\end{aligned}
$$

where $\varphi_{i}(t)=\min \left\{\varsigma_{i}(t), \eta_{i}(t)\right\},(i=1,2, \cdots, q)$ is same as (14). Furthermore, from the system (2), we obtain

$$
A\left(t^{+}\right)+I\left(t^{+}\right)=\left(1-p_{i}\right) A(t)+\left(1-\theta_{i}\right) I(t) \geq\left(1-b_{i}\right)(A(t)+I(t))
$$

where $b_{i}=\max \left\{p_{i}, \theta_{i}\right\},(i=1,2, \cdots, q)$ can be seen in (14).

Then, we consider the following auxiliary impulsive system:

$$
\left\{\begin{array}{l}
\frac{\mathrm{d} y_{2}(t)}{\mathrm{d} t}=\left[\varphi_{k}(t)\left(\frac{\alpha\left(K-\varepsilon_{2}\right)}{\psi^{*} \varepsilon_{2}+\alpha+\gamma}-\varepsilon_{2}\right)-(\gamma+\mu)\right] y_{2}(t), t \neq t_{k}, k \in N, \\
y_{2}\left(t^{+}\right)=\left(1-b_{k}\right) y_{2}(t), t=t_{k}, k \in N . \\
y_{2}\left(t_{0}^{+}\right)=A_{0}+I_{0}>0 .
\end{array}\right.
$$

By solving the impulsive differential equation, we infer that for

$$
\begin{aligned}
& t_{i-1}+n \omega<t<t_{i}+n \omega,\left(n \geq n_{1}\right), i=1,2, \cdots, q, \\
& y_{2}(t)=\left(A_{0}+I_{0}\right) \sum^{n}\left(\prod_{j=1}^{i-1}\left(1-b_{j}\right)\right) \exp \left[\left(\frac{\alpha\left(K-\varepsilon_{2}\right)}{\psi^{*} \varepsilon_{2}+\alpha+\gamma}-\varepsilon_{2}\right)\right. \\
&\left.\cdot\left(\sum_{j=1}^{i-1} \int_{t_{j-1}}^{t_{j}} \varphi_{j}(t) \mathrm{d} t+\int_{t_{i-1}+n \omega}^{t} \varphi_{j}(t) \mathrm{d} t\right)-(\gamma+\mu)\left(t-t_{0}-n \omega\right)\right] .
\end{aligned}
$$

Thus, from (15) and (19), we get

$$
y_{2}(t) \rightarrow+\infty, \text { as } n \rightarrow+\infty,
$$

that is to say, as $t \rightarrow+\infty$, we have $y_{2}(t) \rightarrow+\infty$. By the comparison theorem, we have $\lim _{t \rightarrow+\infty}(A(t)+I(t))=+\infty$, which is a contradiction to $0<A(t)+I(t)<\varepsilon_{2}$. Thus the claim is proved.

By the claim, we need to consider the following two possible cases. The proven method is same as to [23]. 
Case 1. $A(t)+I(t)>\varepsilon_{2}$ for all large $t$;

Case 2. $A(t)+I(t)$ oscillates about $\varepsilon_{2}$ for all large $t$.

Obviously, the conclusion is evident in Case 1 . For Case 2, set $\underline{t}$ and $\bar{t}>t^{3}$ be large enough such that

$$
A(\underline{t})+I(\underline{t}) \geq \varepsilon_{2}, A(\bar{t})+I(\bar{t})=\varepsilon_{2} \text {, and } A(t)+I(t)<\varepsilon_{2} \text {, for } t \in(\underline{t}, \bar{t}) .
$$

There are two possible subcases for $\underline{t}$.

Subcase (I). If $\underline{t}=t_{i}+n \omega$ ( $n$ is a nonnegative integer and $i=1,2, \cdots, q$ ), then $A(\underline{t})+I(\underline{t})>\varepsilon_{2}$ and $\left(1-b_{i}\right) \varepsilon_{2}<\left(1-b_{i}\right)(A(\underline{t})+I(\underline{t})) \leq A\left(\underline{t}^{+}\right)+I\left(\underline{t}^{+}\right)<\varepsilon_{2}$, where $b_{i}$ is same as (18). It's easy to know that there have a positive constant $m$, such that $A(t)+I(t) \geq m$, for all $t \in(\underline{t}, \bar{t})$. Then, we consider two possibilities in the size of $\underline{t}$ and $\bar{t}$.

1) If $\bar{t}-\underline{t} \leq n^{*} \omega$, where $n^{*}$ is same as (17), then from system (2), we have

$$
\left\{\begin{aligned}
\frac{\mathrm{d} A(t)}{\mathrm{d} t}+\frac{\mathrm{d} I(t)}{\mathrm{d} t} & =f(t, S, A)+g(t, S, I)-\gamma(A(t)+I(t))-\mu I(t) \\
& \geq-(\mu+\gamma)(A(t)+I(t)), t \neq t_{k}, k \in N, \\
A\left(t^{+}\right)+I\left(t^{+}\right) & \geq\left(1-b_{k}\right)(A(t)+I(t)), t=t_{k}, k \in N .
\end{aligned}\right.
$$

From (20), we get

$$
\begin{aligned}
A(t)+I(t) & \geq\left[\prod_{i=1}^{q}\left(1-b_{i}\right)\right]^{n^{*}+1}(A(\underline{t})+I(\underline{t})) \exp [-(\gamma+\mu)(t-\underline{t}) \\
& \geq\left[\prod_{i=1}^{q}\left(1-b_{i}\right)\right]^{n^{*}+1} \varepsilon_{2} \exp \left[-(\gamma+\mu) n^{*} \omega\right] \triangleq m,
\end{aligned}
$$

for all $t \in[\underline{t}, \bar{t}]$.

2) If $\bar{t}-\underline{t} \geq n^{*} \omega$, in view of the discussion in (i), we have $A(t)+I(t) \geq m$ for all $t \in\left[\underline{t}, \underline{t}+n^{*} \omega\right]$. Next, we show that $A(t)+I(t) \geq m$ for all $t \in\left(\underline{t}+n^{*} \omega, \bar{t}\right]$. Otherwise, there exists a constant $t^{*}>0$ such that $A(t)+I(t) \geq m$, for all $t \in\left[\underline{t}, \underline{t}+t^{*}+n^{*} \omega\right)$, $A\left(\underline{t}+t^{*}+n^{*} \omega\right)+I\left(\underline{t}+t^{*}+n^{*} \omega\right) \geq m$ and $A(t)+I(t)<m$, for $0<t-\left(\underline{t}+t^{*}+n^{*} \omega\right) \ll 1$.

Next, we consider two possibilities separately:

a) For any $k \in N, \underline{t}+t^{*}+n^{*} \omega \neq t_{k}$.

Obviously, system (18) holds on $\left[\underline{t}+n^{*} \omega, \bar{t}\right]$. Then, we can choose a proper $\rho>0$, such that $A\left(\underline{t}+n^{*} \omega+t^{*}\right)+I\left(\underline{t}+n^{*} \omega+t^{*}\right) \geq \rho\left(A_{0}+I_{0}\right) \geq m$. Using the comparison theorem, we have

$$
\begin{aligned}
& A(t)+I(t) \\
& >\left(A\left(\underline{t}+t^{*}+n^{*} \omega\right)+I\left(\underline{t}+t^{*}+n^{*} \omega\right)\right) \\
& \quad \cdot \exp \left[\left(\frac{\alpha\left(K-\varepsilon_{2}\right)}{\psi^{*} \varepsilon_{2}+\gamma+\alpha}-\varepsilon_{1}\right)-(\gamma+\mu)\left(t-\left(\underline{t}+t^{*}+n^{*} \omega\right)\right)\right] \\
& \geq \rho\left(A_{0}+I_{0}\right) \exp \left[\left(\frac{\alpha\left(K-\varepsilon_{2}\right)}{\psi^{*} \varepsilon_{2}+\gamma+\alpha}-\varepsilon_{1}\right)-(\gamma+\mu)\left(t-\left(\underline{t}+t^{*}+n^{*} \omega\right)\right)\right],
\end{aligned}
$$

when $0<t-\left(\underline{t}+t^{*}+n^{*} \omega\right) \ll 1$. 
In addition, (15) implies that

$$
\exp \left[\left(\frac{\alpha\left(K-\varepsilon_{2}\right)}{\psi^{*} \varepsilon_{2}+\gamma+\alpha}-\varepsilon_{2}\right)-(\gamma+\mu)\left(t-\left(\underline{t}+t^{*}+n^{*} \omega\right)\right)\right] \geq 1
$$

Then, we have

$$
\begin{aligned}
& A(t)+I(t) \\
& \geq \rho\left(A_{0}+I_{0}\right) \exp \left[\left(\frac{\alpha\left(K-\varepsilon_{2}\right)}{\psi^{*} \varepsilon_{2}+\gamma+\alpha}-\varepsilon_{2}\right)-(\gamma+\mu)\left(t-\left(\underline{t}+t^{*}+n^{*} \omega\right)\right)\right] \\
& \geq \rho\left(A_{0}+I_{0}\right) \geq m
\end{aligned}
$$

Then $A(t)+I(t) \geq m$, for $0<t-\left(\underline{t}+t^{*}+n^{*} \omega\right) \ll 1$, which is a contradiction. Therefore, $A(t)+I(t) \geq m$ for all $t \in[\underline{t}, \bar{t}]$.

b) We have a $k \in N$ such that $\underline{t}+t^{*}+n^{*} \omega=t_{k}$. The proof of (b) is same as to (a), we omit it.

Subcase (II). If for any $k \in N, \underline{t} \neq t_{k}$, then $A(\underline{t})+I(\underline{t})=\varepsilon_{2}$. Using the same methods of subcase (I), it is easy to get a positive constant $m$, such that $A(t)+I(t) \geq m$, for all $t \in[\underline{t}, \bar{t}]$.

Thus, we get that $A(t)+I(t) \geq m$ for all $t \in[\underline{t}, \bar{t}]$. Since the interval $[\underline{t}, \bar{t}]$ is chosen in an arbitrary way, we have that $A(t)+I(t) \geq m$ for all large $t$.

From the above analysis, $m$ is independent of the positive solution of model (2), and we have proved that the solution of system (2) satisfies $A(t)+I(t) \geq m$ for sufficiently large $t$, that is to say, $\liminf _{t \rightarrow+\infty}(A(t)+I(t)) \geq m$. We can easily see that, there exists a positive constant $S_{*}$, such that $\liminf _{t \rightarrow+\infty} S(t) \geq S_{*}$. Therefore, the permanence of system (2) is proved.

\section{Conclusion}

In this paper, we studied a pine wilt disease epidemic model with general nonlinear incidence rate and pulse rouging strategy. According to the above analysis of the systems, we know that the disease-free equilibrium is globally attractive determined by the basic reproduction number $R_{1}<1$, and in such a case, the endemic equilibrium does not exist. In Section 4, we proved that the disease uniformly persists only if $R_{2}>1$.

\section{Acknowledgements}

The research has been supported by the Science and Technology Plan Projects of Jiangxi Provincial Education Department (GJJ191443, GJJ181359), Guidance Project of Ji'an Science and Technology Bureau.

\section{Conflicts of Interest}

The authors declare no conflicts of interest regarding the publication of this paper.

\section{References}

[1] Kiyohara, T. and Tokushige, Y. (1971) Inoculation Experiments of a Nematode, Bursaphelenchus sp., onto Pine Trees. Journal of the Japanese Forestry Society, 53, 
210-218. (In Japanese)

[2] Mamiya, Y. and Kiyohara, T. (1972) Description of Bursaphelenchus lignicolus n. sp. (Nematoda: Aphelenchoididae) from Pine Wood and Histopathology of Nematode-Infested Trees. Nematologica, 18, 120-124. https://doi.org/10.1163/187529272X00296

[3] Nickle, W.A.R., Gloden, A.M., et al. (1981) On the Taxonomy and Morphology of the Pine Wood Nematode, Bursaphelenchus xylophilus (Steiner and Buhrer 1934) Nickle 1970. Journal of Nematologica, 13, 385-392.

[4] Mamiya, Y. and Enda, N. (1972) Transmission of Bursaphelenchus lignicolus (Nematoda: Aphelenchoididae) by Monochamus alternatus (Coleoptera: Cerambycidae). Nematologica, 18, 159-162. https://doi.org/10.1163/187529272X00395

[5] Morimoto, K. and Iwasaki, A. (1972) Role of Monochamus alternatus (Coleoptera: Cerambycidae) as a Vector of Bursaphelenchus lignicolus (Nematoda: Aphelenchoididae). Journal of the Japanese Forestry Society, 54, 177-183. (In Japanese)

[6] Wingfield, M.J. and Blanchette, R.B. (1983) The Pine-Wood Nematode, Bursaphelenchus xylophilus, in Minnesota and Wisconsin: Insect Associates and Transmission Studies. Canadian Journal of Forest Research, 13, 1068-1076.

https://doi.org/10.1139/x83-143

[7] Sato, H., Sakuyama, T. and Kobayashi, M. (1987) Transmission of Bursaphelenchus xylophilus (Steiner et Buhrer) Nickle (Nematoda, Aphelenchoididae) by Monochamus saltuarius (Gebler, Coleoptera, Cerambycidae). Journal of the Japanese Forestry Society, 69, 492-496. (In Japanese)

[8] Linit, M.J. (1988) Nematode-Vector Relationships in the Pine Wilt Disease System. Journal of Nematology, 20, 227-235.

[9] Arakawa, Y. and Togashi, K. (2002) Newly Discovered Transmission Pathway of Bursaphelenchus xylophilus from Males of the Beetle Monochamus alternatus to Pinus densiflora Trees via Oviposition Wounds. The Journal of Nematology, 34, 396-404.

[10] Mota, M.M., Braasch, H., et al. (1999) First Report of Bursaphelenchus xylophilus in Portugal and in Europe. Nematology, 1, 727-734. https://doi.org/10.1163/156854199508757

[11] Lee, K.S. (2014) Stability Analysis and Optimal Control Strategy for Prevention of Pine Wilt Disease. Abstract and Applied Analysis, 2014, Article ID: 182680. https://doi.org/10.1155/2014/182680

[12] Shi, X.Y. and Song, G.H. (2013) Analysis of the Mathematical Model for the Spread of Pine Wilt Disease. Journal of Applied Mathematics, 2013, Article ID: 184054. https://doi.org/10.1155/2013/184054

[13] Ozair, M. (2014) Analysis of Pine Wilt Disease Model with Nonlinear Incidence and Horizontal Transmission. Journal of Applied Mathematics, 2014, Article ID: 204241. https://doi.org/10.1155/2014/204241

[14] Capasso, V. and Serio, G. (1978) A Generalization of the Kermack-McKendrick Deterministic Epidemic Model. Mathematical Biosciences, 42, 43-61.

https://doi.org/10.1016/0025-5564(78)90006-8

[15] Liu, W.M., Hethcote, H.W. and Levin, S.A. (1987) Dynamical Behavior of Epidemiological Models with Nonlinear Incidence Rates. Journal of Mathematical Biology, 25, 359-380. https://doi.org/10.1007/BF00277162

[16] Teng, Z. and Wang, L. (2016) Persistence and Extinction for a Class of Stochastic SIS Epidemic Models with Nonlinear Incidence Rate. Physica A, 451, 507-518. 
https://doi.org/10.1016/j.physa.2016.01.084

[17] Lee, K.S. and Kim, D. (2013) Global Dynamics of a Pine Wilt Disease Transmission Model with Nonlinear Incidence Rates. Applied Mathematical Modelling, 37, 4561-4569. https://doi.org/10.1016/j.apm.2012.09.042

[18] Khan, M.A., et al. (2017) Mathematical Modeling and Stability Analysis of Pine Wilt Disease with Optimal Control. Scientific Reports, 7, Article No. 3115. https://doi.org/10.1038/s41598-017-03179-w

[19] Khan, M.A., Khan, R., et al. (2018) A Mathematical Analysis of Pine Wilt Disease with Variable Population Size and Optimal Control Strategies. Chaos, Solitons and Fractals, 108, 205-217. https://doi.org/10.1016/j.chaos.2018.02.002

[20] Khan, M.A., Shah, K., et al. (2018) Mathematical Modeling Approach to the Transmission Dynamics of Pine Wilt Disease with Saturated Incidence Rate. International Journal of Biomaterials, 11, Article ID: 1850035. https://doi.org/10.1142/S1793524518500353

[21] Yu, D., Gao, S.J., et al. (2016) Pulse Vaccination Strategy in an SIVS Epidemic Model with General Nonlinear Incidence Rate. Advances in Analysis, 1, 80-88.

[22] Gao, S., Chen, L., Nieto, J.J., et al. (2006) Analysis of a Delayed Epidemic Model with Pulse Vaccination and Saturation Incidence. Vaccine, 24, 6037-6045. https://doi.org/10.1016/j.vaccine.2006.05.018

[23] Wang, J., Zhang, X., Wang, J., et al. (2019) Dynamics of a Nonautonomous Plant Disease Model with General Nonlinear Incidence Rate and Time-Varying Impulse. Journal of Applied Mathematics and Physics, 7, 2518-2530.

https://doi.org/10.4236/jamp.2019.710171 